\title{
Dirichlet spaces with superharmonic weights and de Branges-Rovnyak spaces
}

\author{
O. El-Fallah, K. Kellay, H. Klaja, J. Mashreghi and T. Ransford
}

\begin{abstract}
We consider Dirichlet spaces with superharmonic weights. This class contains both the harmonic weights and the power weights. Our main result is a characterization of the Dirichlet spaces with superharmonic weights that can be identified as de Branges-Rovnyak spaces. As an application, we obtain the dilation inequality

$$
\mathcal{D}_{\omega}\left(f_{r}\right) \leq \frac{2 r}{1+r} \mathcal{D}_{\omega}(f) \quad(0 \leq r<1),
$$

where $\mathcal{D}_{\omega}$ denotes the Dirichlet integral with superharmonic weight $\omega$, and $f_{r}(z):=f(r z)$ is the $r$-dilation of the holomorphic function $f$.
\end{abstract}

Mathematics Subject Classification (2010). Primary 31C25, 46E22; Secondary $30 \mathrm{H} 10$.

Keywords. Dirichlet space; superharmonic; de Branges-Rovnyak space.

\section{Introduction}

Let $\mathbb{D}$ be the open unit disk, let $d A$ be normalized area measure on $\mathbb{D}$, and let $\operatorname{Hol}(\mathbb{D})$ be the space of holomorphic functions on $\mathbb{D}$. Given a non-negative function $\omega \in L^{1}(\mathbb{D})$ and $f \in \operatorname{Hol}(\mathbb{D})$, we define

$$
\mathcal{D}_{\omega}(f):=\int_{\mathbb{D}}\left|f^{\prime}(z)\right|^{2} \omega(z) d A(z) .
$$

The weighted Dirichlet space $\mathcal{D}_{\omega}$ is the set of $f \in \operatorname{Hol}(\mathbb{D})$ with $\mathcal{D}_{\omega}(f)<\infty$. Obviously, if $\omega \equiv 1$, then $\mathcal{D}_{\omega}$ is just the classical Dirichlet space $\mathcal{D}$.

In this article we shall be mainly interested in the case where $\omega$ is a superharmonic weight. This class of weights was introduced by Aleman [1].

El-Fallah supported by CNRST URAC/03 \& Académie HassanII des sciences et techniques.

Kellay supported by UMI-CRM.

Mashreghi supported by NSERC.

Ransford supported by NSERC and the Canada research chairs program. 
For such $\omega$, we automatically have $\omega \in L^{1}(\mathbb{D})$, and, provided that $\omega \neq \equiv 0$, we also have $\mathcal{D}_{\omega} \subset H^{2}$, the Hardy space. It is customary to define

$$
\|f\|_{\mathcal{D}_{\omega}}^{2}:=\|f\|_{H^{2}}^{2}+\mathcal{D}_{\omega}(f) \quad\left(f \in \mathcal{D}_{\omega}\right),
$$

making $\mathcal{D}_{\omega}$ a Hilbert space.

The class of superharmonic weights includes two important subclasses:

- the power weights $\omega(z):=\left(1-|z|^{2}\right)^{\alpha}(0 \leq \alpha \leq 1)$, which form a scale linking the classical Dirichlet space $(\alpha=0)$ to the Hardy space $(\alpha=1)$.

- the harmonic weights, introduced by Richter [11] in connection with his analysis of shift-invariant subspaces of the classical Dirichlet space.

Superharmonic weights have the important property that the dilates of $f_{r}(z):=f(r z)$ of each function $f \in \mathcal{D}_{\omega}$ satisfy

$$
\mathcal{D}_{\omega}\left(f_{r}\right) \leq C \mathcal{D}_{\omega}(f) \quad(0 \leq r<1),
$$

where $C$ is an absolute constant. From this, it is not hard to deduce that $\left\|f_{r}-f\right\|_{\mathcal{D}_{\omega}} \rightarrow 0$ as $r \rightarrow 1^{-}$, and that polynomials are dense in $\mathcal{D}_{\omega}$.

Inequality (1.1) was first proved by Richter and Sundberg [12] in the case where $\omega$ is harmonic, with $C=4$. It was generalized to superharmonic weights by Aleman [1], with an improved constant $C=5 / 2$. For harmonic weights, the constant was further improved to $C=1$ by Sarason 14 .

The basis of Sarason's method was to show that, if $\omega=P_{\zeta}$ (the Poisson kernel at a point $\zeta \in \mathbb{T}$ ), then it is possible to identify $\mathcal{D}_{\omega}$ as a de BrangesRovnyak space $\mathcal{H}(b)$, the norms being identical (we shall define $\mathcal{H}(b)$ later). Working within $\mathcal{H}(b)$, one can deduce that (1.1) holds with $C=1$, at least for these special $\omega$. Inequality (1.1) for general harmonic $\omega$ then follows easily by using an averaging argument.

It turns out that Sarason's construction has a sort of converse. The only harmonic weights $\omega$ for which $\mathcal{D}_{\omega}$ can be identified as a de Branges-Rovnyak space are multiples of $P_{\zeta}$, where $\zeta \in \mathbb{T}$. This was proved in 2]. (One can also study a weaker notion of 'identified', where the spaces $\mathcal{D}_{\omega}$ and $\mathcal{H}(b)$ are allowed to carry different norms. This appears to be more subtle: see [3].)

Our purpose in this paper is twofold. First, we shall extend Sarason's result by exhibiting a new family of superharmonic weights $\omega$ for which $\mathcal{D}_{\omega}$ can be identified as a de Branges-Rovnyak space $\mathcal{H}(b)$. We shall deduce from this that the inequality (1.1) holds with $C=1$ for all superharmonic weights. Second, we shall prove a converse result, characterizing those superharmonic weights $\omega$ for which $\mathcal{D}_{\omega}$ is equal to an $\mathcal{H}(b)$. We shall also consider what happens for more general weights.

\section{Superharmonic weights}

Let $\omega$ be a positive superharmonic function on $\mathbb{D}$.

By standard results from potential theory, $\omega$ is locally integrable on $\mathbb{D}$, and $\left(1 / r^{2}\right) \int_{|z| \leq r} \omega d A$ is a decreasing function of $r$ for $0<r<1$. It follows that $\omega \in L^{1}(\mathbb{D})$, so it is admissible as a Dirichlet weight. 
Now suppose that $\omega \not \equiv 0$. We shall show that $\mathcal{D}_{\omega} \subset H^{2}$. By the minimum principle $\omega(z)>0$ for all $z \in \mathbb{D}$ (possibly infinite at some points). By lower semicontinuity, $m:=\inf _{|z| \leq 1 / e} \omega(z)$ is attained and therefore $m>0$. By the minimum principle again, $\omega(z) \geq m \log (1 /|z|)$ for all $z$ with $1 / e<|z|<1$. Since the Dirichlet space with weight $\log (1 /|z|)$ is just $H^{2}$, it follows easily that $\mathcal{D}_{\omega} \subset H^{2}$, as claimed.

It thus makes sense to define the norm $\|\cdot\|_{\mathcal{D}_{\omega}}$ on $\mathcal{D}_{\omega}$ by

$$
\|f\|_{\mathcal{D}_{\omega}}^{2}:=\|f\|_{H^{2}}^{2}+\mathcal{D}_{\omega}(f) \quad\left(f \in \mathcal{D}_{\omega}\right) .
$$

With respect to this norm, $\mathcal{D}_{\omega}$ is a Hilbert space.

We shall make extensive use of the following representation formula for positive superharmonic functions $\omega$ on $\mathbb{D}$. Given such an $\omega$, there exists a unique positive finite Borel measure $\mu$ on $\overline{\mathbb{D}}$ such that, for all $z \in \mathbb{D}$,

$$
\omega(z)=\int_{\mathbb{D}} \log \left|\frac{1-\bar{\zeta} z}{\zeta-z}\right| \frac{2}{1-|\zeta|^{2}} d \mu(\zeta)+\int_{\mathbb{T}} \frac{1-|z|^{2}}{|\zeta-z|^{2}} d \mu(\zeta) .
$$

(This is the usual decomposition of $\omega$ as a potential plus a harmonic function.) We then write $\mathcal{D}_{\mu}$ for $\mathcal{D}_{\omega}$, and further $\mathcal{D}_{\zeta}$ for $\mathcal{D}_{\delta_{\zeta}}$. Thus, for $f \in \operatorname{Hol}(\mathbb{D})$,

$$
\mathcal{D}_{\zeta}(f)= \begin{cases}\int_{\mathbb{D}} \log \left|\frac{1-\bar{\zeta} z}{\zeta-z}\right| \frac{2}{1-|\zeta|^{2}}\left|f^{\prime}(z)\right|^{2} d A(z), & \zeta \in \mathbb{D}, \\ \int_{\mathbb{D}} \frac{1-|z|^{2}}{|\zeta-z|^{2}}\left|f^{\prime}(z)\right|^{2} d A(z), & \zeta \in \mathbb{T} .\end{cases}
$$

For general $\mu$, we can recover $\mathcal{D}_{\mu}(f)$ from $\mathcal{D}_{\zeta}(f)$ via Fubini's theorem:

$$
\mathcal{D}_{\mu}(f)=\int_{\overline{\mathbb{D}}} \mathcal{D}_{\zeta}(f) d \mu(\zeta)
$$

Finally, we shall need the following Douglas-type formula for $\mathcal{D}_{\zeta}(f)$.

Theorem 2.1. Let $f \in H^{2}$ and let $\zeta \in \overline{\mathbb{D}}$. If $\zeta \in \mathbb{D}$, then

$$
\mathcal{D}_{\zeta}(f)=\frac{1}{2 \pi} \int_{\mathbb{T}}\left|\frac{f(\lambda)-f(\zeta)}{\lambda-\zeta}\right|^{2}|d \lambda| .
$$

If $\zeta \in \mathbb{T}$ and $f(\zeta):=\lim _{r \rightarrow 1^{-}} f(r \zeta)$ exists, then the same formula holds. Otherwise $\mathcal{D}_{\zeta}(f)=\infty$.

For a proof, and more background on superharmonic weights, see [1].

\section{De Branges-Rovnyak spaces}

The de Branges-Rovnyak spaces are a family of (not necessarily closed) subspaces $\mathcal{H}(b)$ of $H^{2}$, parametrized by elements $b$ of the closed unit ball of $H^{\infty}$. They were introduced by de Branges and Rovnyak in the appendix of [4] and further studied in 5. For background information we refer to the books of de Branges and Rovnyak [5], Sarason [13], and the forthcoming monograph of Fricain and Mashreghi [9]. 
Given $\psi \in L^{\infty}(\mathbb{T})$, we define the Toeplitz operator $T_{\psi}: H^{2} \rightarrow H^{2}$ by

$$
T_{\psi} f:=P_{+}(\psi f) \quad\left(f \in H^{2}\right),
$$

where $P_{+}: L^{2}(\mathbb{T}) \rightarrow H^{2}$ is the orthogonal projection of $L^{2}(\mathbb{T})$ onto $H^{2}$. Clearly $T_{\psi}$ is a bounded operator on $H^{2}$, and its adjoint is $T_{\bar{\psi}}$.

Given $b \in H^{\infty}$ with $\|b\|_{H^{\infty}} \leq 1$, the de Branges-Rovnyak space $\mathcal{H}(b)$ is defined as the image of $H^{2}$ under the operator $\left(I-T_{b} T_{\bar{b}}\right)^{1 / 2}$. We put a norm on $\mathcal{H}(b)$ making $\left(I-T_{b} T_{\bar{b}}\right)^{1 / 2}$ a partial isometry from $H^{2}$ onto $\mathcal{H}(b)$, namely

$$
\left.\left\|\left(I-T_{b} T_{\bar{b}}\right)^{1 / 2} f\right\|_{\mathcal{H}(b)}:=\|f\|_{H^{2}} \quad\left(f \in H^{2} \ominus \operatorname{ker}\left(I-T_{b} T_{\bar{b}}\right)^{1 / 2}\right)\right) .
$$

We have defined $\mathcal{H}(b)$ as in Sarason's book 13 . The original definition of de Branges and Rovnyak, based on the notion of complementary space, is different but equivalent. An explanation of the equivalence can be found in [13. pp.7-8]. A third approach is to start from the positive kernel

$$
k_{w}^{b}(z):=\frac{1-\overline{b(w)} b(z)}{1-\bar{w} z} \quad(z, w \in \mathbb{D}),
$$

and to define $\mathcal{H}(b)$ as the reproducing kernel Hilbert space associated with this kernel.

The theory of $\mathcal{H}(b)$-spaces is pervaded by a fundamental dichotomy, namely whether $b$ is or is not an extreme point of the closed unit ball of $H^{\infty}$. This is illustrated by following result.

Theorem 3.1. Let $b \in H^{\infty}$ with $\|b\|_{H^{\infty}} \leq 1$. The following are equivalent:

(i) $b$ is a non-extreme point of the closed unit ball of $H^{\infty}$;

(ii) $\log \left(1-|b|^{2}\right) \in L^{1}(\mathbb{T})$;

(iii) $\mathcal{H}(b)$ contains all functions holomorphic in a neighborhood of $\overline{\mathbb{D}}$.

Furthermore, if $b$ is non-extreme, then the polynomials are dense in $\mathcal{H}(b)$.

Proof. The equivalence between (i) and (ii) is proved in [6, Theorem 7.9]. That (i) implies (iii) is proved in [13, §IV-6], and that (iii) implies (i) follows from [13, $\S \mathrm{V}-1]$. Finally, the density of polynomials when $b$ is non-extreme is proved in [13, $§ I \mathrm{I}-3]$; a constructive proof of this result is given in [7].

Henceforth we shall simply say that $b$ is 'extreme' or 'non-extreme', it being understood that this relative to the closed unit ball of $H^{\infty}$.

From the equivalence between (i) and (ii), it follows that, if $b$ is nonextreme, then there exists a unique outer function $a$ such that $a(0)>0$ and $|a|^{2}+|b|^{2}=1$ a.e. on $\mathbb{T}$ (see [13, §IV-1]). We shall call $(b, a)$ a pair. The following result gives a useful characterization of $\mathcal{H}(b)$ in this case.

Theorem 3.2 ([13, $\S \mathrm{IV}-1])$. Let $b$ be non-extreme, let $(b, a)$ be a pair and let $f \in H^{2}$. Then $f \in \mathcal{H}(b)$ if and only if $T_{\bar{b}} f \in T_{\bar{a}}\left(H^{2}\right)$. In this case, there exists a unique function $f^{+} \in H^{2}$ such that $T_{\bar{b}} f=T_{\bar{a}} f^{+}$, and

$$
\|f\|_{\mathcal{H}(b)}^{2}=\|f\|_{H^{2}}^{2}+\left\|f^{+}\right\|_{H^{2}}^{2} \text {. }
$$


Given a pair $(b, a)$, the function $\phi:=b / a$ is the quotient of two functions in $H^{\infty}$, the denominator being outer. In other words, $\phi \in N^{+}$, the Smirnov class. Conversely, given $\phi \in N^{+}$, we can write $\phi=b / a$, where $a, b \in H^{\infty}$ and $a$ is outer. Multiplying $a$ and $b$ by an appropriately chosen outer function, we may further ensure that $|a|^{2}+|b|^{2}=1$ a.e. on $\mathbb{T}$ and that $a(0)>0$, in other words, that $(b, a)$ is a pair. Then $a$ and $b$ are uniquely determined. There is thus a bijection $b \leftrightarrow \phi$ between non-extreme functions $b$ and elements $\phi$ of the Smirnov class. Note that $b$ and $\phi$ have the same inner factor. Also $\phi$ is bounded if and only if $\|b\|_{H^{\infty}}<1$. In this case, $\left(I-T_{b} T_{\bar{b}}\right)$ is an invertible operator on $H^{2}$, and consequently $\mathcal{H}(b)=H^{2}$ as vector spaces, although the norms may be different.

\section{Extension of Sarason's theorem}

Our goal in this section is to establish the following theorem and examine one of its consequences.

Theorem 4.1. Let $\zeta \in \overline{\mathbb{D}}$, and let $b \in H^{\infty}$ be the function corresponding to $\phi(z):=z /(1-\bar{\zeta} z)$. Then $\mathcal{D}_{\zeta}=\mathcal{H}(b)$ with equality of norms.

Remarks. (1) This theorem extends Sarason's result [14], which is the special case $\zeta \in \mathbb{T}$.

(2) A computation shows that, with $\phi(z)=z /(1-\bar{\zeta} z)$, we have

$$
b(z)=\frac{z}{A-B z} \quad \text { and } \quad a(z)=\frac{1-\bar{\zeta} z}{A-B z}
$$

where

$$
A:=\left(\frac{2+|\zeta|^{2}+\sqrt{4+|\zeta|^{4}}}{2}\right)^{1 / 2} \text { and } B:=\left(\frac{2+|\zeta|^{2}-\sqrt{4+|\zeta|^{4}}}{2}\right)^{1 / 2} \frac{\bar{\zeta}}{|\zeta|}
$$

However, we do not need the precise formulas for $b$ and $a$ in what follows.

Proof. As the result is already known for $\zeta \in \mathbb{T}$, we shall concentrate on the case $\zeta \in \mathbb{D}$. In this case, $\mathcal{D}_{\zeta}$ and $\mathcal{H}_{b}$ are both equal to $H^{2}$ as vector spaces, with equivalent norms. For $\mathcal{D}_{\zeta}$ this follows from Theorem 2.1, and for $\mathcal{H}(b)$ we already observed this to be true whenever $\phi$ is bounded. The content of the theorem is that the norms on $\mathcal{D}_{\zeta}$ and $\mathcal{H}(b)$ are in fact identical.

For $w \in \mathbb{D}$, let $k_{w}$ denote the Cauchy kernel, namely

$$
k_{w}(z):=\frac{1}{1-\bar{w} z} \quad(z \in \mathbb{D}) .
$$

It is enough to prove that $\langle f, g\rangle_{\mathcal{D}_{\zeta}}=\langle f, g\rangle_{\mathcal{H}(b)}$ when $f$ and $g$ are finite linear combinations of Cauchy kernels, since such $f, g$ are dense in $H^{2}$. By sesquilinearity, this reduces to checking that $\left\langle k_{w_{1}}, k_{w_{2}}\right\rangle_{\mathcal{D}_{\zeta}}=\left\langle k_{w_{1}}, k_{w_{2}}\right\rangle_{\mathcal{H}(b)}$ for all $w_{1}, w_{2} \in \mathbb{D}$. As both sides of this last equation are holomorphic in $w_{2}$ and antiholomorphic in $w_{1}$, it is sufficient to prove it in the case when $w_{1}=w_{2}$. Thus we need to show that $\left\|k_{w}\right\|_{\mathcal{D}_{\zeta}}^{2}=\left\|k_{w}\right\|_{\mathcal{H}(b)}^{2}$ for all $w \in \mathbb{D}$. 
By (2.1) and (3.1), this amounts to verifying that $\mathcal{D}_{\zeta}\left(k_{w}\right)=\left\|k_{w}^{+}\right\|_{H^{2}}^{2}$ for all $w \in \mathbb{D}$, which we now proceed to do.

On the one hand, by Theorem 2.1, we have

$$
\mathcal{D}_{\zeta}\left(k_{w}\right)=\frac{1}{2 \pi} \int_{\mathbb{T}}\left|\frac{k_{w}(\lambda)-k_{w}(\zeta)}{\lambda-\zeta}\right|^{2}|d \lambda|=\left\|\frac{\bar{w}}{1-\bar{w} \zeta} k_{w}\right\|_{H^{2}}^{2} .
$$

The Cauchy kernel is the reproducing kernel for $H^{2}$, so $\left\langle f, k_{w}\right\rangle_{H^{2}}=f(w)$ for all $f \in H^{2}$, and in particular $\left\|k_{w}\right\|_{H^{2}}^{2}=k_{w}(w)=1 /\left(1-|w|^{2}\right)$. Hence

$$
\mathcal{D}_{\zeta}\left(k_{w}\right)=\frac{|w|^{2}}{|1-\bar{w} \zeta|^{2}\left(1-|w|^{2}\right)} .
$$

On the other hand, by a standard property of adjoints of multiplication operators acting on reproducing kernels, we have $T_{\bar{h}} k_{w}=\overline{h(w)} k_{w}$ for all $h \in H^{\infty}$ and $w \in \mathbb{D}$. In particular this is true when $h=b$ and when $h=a$, where $(b, a)$ is the pair with $b / a=\phi$. In the notation of Theorem 3.2 it follows that $k_{w}^{+}=\overline{\phi(w)} k_{w}$, whence

$$
\left\|k_{w}^{+}\right\|_{H^{2}}^{2}=|\phi(w)|^{2}\left\|k_{w}\right\|_{H^{2}}^{2}=\frac{|w|^{2}}{|1-\bar{w} \zeta|^{2}\left(1-|w|^{2}\right)} .
$$

Thus $\left\|k_{w}^{+}\right\|_{H^{2}}^{2}=\mathcal{D}_{\zeta}\left(k_{w}\right)$ for all $w \in \mathbb{D}$, as desired.

Using the same basic idea as in [14, we can use this theorem to deduce that (1.1) holds for all superharmonic weights $\omega$ with constant $C=1$. In fact, just as in 14, we have the following even stronger result.

Theorem 4.2. Let $\mu$ be a finite positive measure on $\overline{\mathbb{D}}$ and let $f \in \mathcal{D}_{\mu}$. Then

$$
\mathcal{D}_{\mu}\left(f_{r}\right) \leq \frac{2 r}{1+r} \mathcal{D}_{\mu}(f) \quad(0 \leq r<1) .
$$

Proof. We prove the result for $\mu=\delta_{\zeta}(\zeta \in \overline{\mathbb{D}})$. The general case follows by integrating up and using (2.3).

By Theorem 4.1, we have $\mathcal{D}_{\zeta}(f)=\left\|f^{+}\right\|_{\mathcal{H}_{b}}^{2}$, where $(b, a)$ is the pair for which $\phi(z):=b(z) / a(z)=z /(1-\bar{\zeta} z)$. So we need to show that, with this choice of $\phi$, we have

$$
\left\|\left(f_{r}\right)^{+}\right\|_{H^{2}}^{2} \leq \frac{2 r}{1+r}\left\|f^{+}\right\|_{H^{2}}^{2} .
$$

Given $h \in H^{2}$, we have

$$
\left\langle\left(f_{r}\right)^{+}, a h\right\rangle_{H^{2}}=\left\langle f_{r}, b h\right\rangle_{H^{2}}=\left\langle f, b_{r} h_{r}\right\rangle_{H^{2}}=\left\langle f^{+},\left(\phi_{r} / \phi\right) a_{r} h_{r}\right\rangle_{H^{2}},
$$

and thus

$$
\left|\left\langle\left(f_{r}\right)^{+}, a h\right\rangle_{H^{2}}\right| \leq\left\|f^{+}\right\|_{H^{2}}\left\|\phi_{r} / \phi\right\|_{H^{\infty}}\|a h\|_{H^{2}} .
$$

As $a$ is an outer function, $a H^{2}$ is dense in $H^{2}$, and so

$$
\left\|\left(f_{r}\right)^{+}\right\|_{H^{2}} \leq\left\|\phi_{r} / \phi\right\|_{H^{\infty}}\left\|f^{+}\right\|_{H^{2}} .
$$

Finally, an elementary computation shows that

$$
\left\|\phi_{r} / \phi\right\|_{H^{\infty}}=r(1+|\zeta|) /(1+r|\zeta|) \leq 2 r /(1+r),
$$

whence the result. 


\section{A converse result}

Theorem 4.1 furnishes a list of couples $(\mu, b)$ for which $\mathcal{D}_{\mu}=\mathcal{H}(b)$. In this section we prove a converse, which shows that, apart from scalar multiples taken in a natural sense, these are the only such couples. It generalizes a result of [2], where it was proved in the case when $\mu$ is a measure on $\mathbb{T}$.

Note that we may assume from the outset that $b$ is a non-extreme point of the closed unit ball of $H^{\infty}$. Indeed, if we are to have $\mathcal{D}_{\mu}=\mathcal{H}(b)$, then, since $\mathcal{D}_{\mu}$ contains all functions holomorphic on a neighborhood of $\overline{\mathbb{D}}$, the same must be true of $\mathcal{H}(b)$. By Theorem 3.1, this entails that $b$ is non-extreme.

Theorem 5.1. Let $\mu$ be a finite positive measure on $\overline{\mathbb{D}}$ with $\mu \neq \equiv$. Let $(b, a)$ be pair and let $\phi:=b / a$. Then $\mathcal{D}_{\mu}=\mathcal{H}(b)$ with equality of norms if and only if there exist $\zeta \in \overline{\mathbb{D}}$ and $\alpha \in \mathbb{C} \backslash\{0\}$ such that

$$
\mu=|\alpha|^{2} \delta_{\zeta} \quad \text { and } \quad \phi(z)=\alpha z /(1-\bar{\zeta} z) .
$$

Proof. The 'if' part follows directly from Theorem 4.1. For the 'only if', we observe that, if we have equality of norms, then $\left\|f^{+}\right\|_{H^{2}}^{2}=\mathcal{D}_{\mu}(f)$ for all $f$ in the space, in particular for $f=k_{w}$, the Cauchy kernels. Performing similar calculations to those in the proof of Theorem 4.1 we obtain the relation

$$
|\phi(w)|^{2}=|w|^{2} \int_{\overline{\mathbb{D}}} \frac{d \mu(z)}{|1-z \bar{w}|^{2}} \quad(w \in \mathbb{D}) .
$$

In particular $\phi(0)=0$. If we write $\phi(w) / w$ as a Taylor series, substitute it into the formula above, expand in powers of $w$ and $\bar{w}$, and equate coefficients, then we end up with the following relation between the moments of $\mu$ :

$$
\mu(\overline{\mathbb{D}}) \int_{\overline{\mathbb{D}}} z^{n} \bar{z}^{m} d \mu(z)=\int_{\overline{\mathbb{D}}} z^{n} d \mu(z) \int_{\overline{\mathbb{D}}} \bar{z}^{m} d \mu(z) \quad(m, n \geq 0) .
$$

In particular, we have $\mu(\overline{\mathbb{D}})\left(\int|z|^{2} d \mu\right)=\left(\int z d \mu\right)\left(\int \bar{z} d \mu\right)$. This can be rewritten as $\int|z-\zeta|^{2} d \mu(z)=0$, where $\zeta:=\left(\int z d \mu\right) / \mu(\overline{\mathbb{D}}) \in \overline{\mathbb{D}}$. It follows that $\mu=c \delta_{\zeta}$ for some $c>0$. Substituting this back into formula (5.1), we find that $|\phi(w)|^{2}=c|w|^{2} /|1-\bar{\zeta} w|^{2}$, and so $\phi(w)=\alpha w /(1-\bar{\zeta} w)$, where $\alpha$ is a complex constant with $|\alpha|^{2}=c$. This completes the proof.

\section{More general weights}

We have identified those superharmonic weights $\omega$ for which $\mathcal{D}_{\omega}$ is equal to a de Branges-Rovnyak space $\mathcal{H}(b)$. What about more general weights? In this section we obtain some results in this direction, leading to questions that we think are interesting in their own right.

We begin with a result about which functions $b$ can arise in this context.

Theorem 6.1. Let $b$ be in the closed unit ball of $H^{\infty}$. If there exists a weight $\omega$ with $0<\|\omega\|_{L^{1}(\mathbb{D})}<\infty$ such that $\mathcal{H}(b)=\mathcal{D}_{\omega}$, with equality of norms, then $b$ is non-extreme and its inner factor is exactly $z$. Consequently the inner factor of the associated function $\phi$ is also $z$. 
Remark. This result is in stark contrast with what happens if we do not insist on equality of norms. See [3, Theorem 4.5].

Proof. That $b$ is non-extreme is proved just as in the remark preceding the proof of Theorem 5.1. We then have $\left\|f^{+}\right\|_{H^{2}}^{2}=\mathcal{D}_{\omega}(f)$ for all $f \in \mathcal{H}(b)$. In particular $f^{+}=0$ if and only if $\mathcal{D}_{\omega}(f)=0$. Now,

$$
f^{+}=0 \Longleftrightarrow T_{\bar{b}} f=0 \Longleftrightarrow f \perp b H^{2} \Longleftrightarrow f \perp b_{i} H^{2},
$$

where $b_{i}$ is the inner factor of $b$. Also, since $\|\omega\|_{L^{1}(\mathbb{D})}>0$, we have

$$
\mathcal{D}_{\omega}(f)=0 \Longleftrightarrow f \text { is a constant } \Longleftrightarrow f \perp z H^{2} .
$$

Combining these remarks, we conclude that $b_{i} H^{2}=z H^{2}$, whence $b_{i}=z$.

The final statement of the theorem is a consequence of the fact, remarked in 33 , that $b$ and $\phi$ have the same inner factor.

We next characterize those weights $\omega$ for which $\mathcal{D}_{\omega}$ is equal to some de Branges-Rovnyak space $\mathcal{H}(b)$. There is no harm in normalizing $\omega$ so that $\|\omega\|_{L^{1}(\mathbb{D})}=1$. To state our result we need to introduce some notation. Given $\psi \in L^{1}(\mathbb{D})$, we denote by $Q \psi$ its Bergman projection, given by

$$
Q \psi(z):=\int_{\mathbb{D}} \frac{\psi(w)}{(1-\bar{w} z)^{2}} d A(w) \quad(z \in \mathbb{D}),
$$

and by $B \psi$ its Berezin transform, defined by

$$
B \psi(z):=\int_{\mathbb{D}} \frac{\left(1-|z|^{2}\right)^{2}}{|1-\bar{w} z|^{4}} \psi(w) d A(w) \quad(z \in \mathbb{D}) .
$$

For further information about these, we refer to [10].

Theorem 6.2. Let $\omega$ be a weight with $\|\omega\|_{L^{1}(\mathbb{D})}=1$. Then there exists $b$ such that $\mathcal{D}_{\omega}=\mathcal{H}(b)$, with equality of norms, if and only if:

(i) $\mathcal{D}_{\omega} \subset H^{2}$,

(ii) polynomials are dense in $\mathcal{D}_{\omega}$, and

(iii) $Q \omega$ is outer and satisfies

$$
\left(1-|z|^{2}\right)|Q \omega(z)|^{2}=B \omega(z) \quad(z \in \mathbb{D}) .
$$

In this case the associated $\phi$ is given by $\phi(z)=c z(Q \omega)(z)$, where $c \in \mathbb{T}$.

Proof. First we prove the 'only if'. If $\mathcal{D}_{\omega}=\mathcal{H}(b)$, then clearly $\mathcal{D}_{\omega} \subset H^{2}$. Also, as remarked in the previous theorem, $b$ is non-extreme, so by Theorem 3.1 polynomials are dense in $\mathcal{H}(b)$, and thus also in $\mathcal{D}_{\omega}$. This proves (i) and (ii). For (iii), note that equality of norms implies $\left\|k_{z}^{+}\right\|_{H^{2}}^{2}=\mathcal{D}_{\omega}\left(k_{z}\right)$ for all $z \in \mathbb{D}$, where once again $k_{z}$ denotes the Cauchy kernel. This yields the identity

$$
\frac{|\phi(z)|^{2}}{1-|z|^{2}}=\int_{\mathbb{D}} \frac{|z|^{2}}{|1-\bar{z} w|^{4}} \omega(w) d A(w) \quad(z \in \mathbb{D}) .
$$

By Theorem 6.1, we have $\phi(z)=z \phi_{o}(z)$, where $\phi_{o}$ is outer. It follows that

$$
\left|\phi_{o}(z)\right|^{2}=\int_{\mathbb{D}} \frac{1-|z|^{2}}{|1-\bar{z} w|^{4}} \omega(w) d A(w) \quad(z \in \mathbb{D}) .
$$


In particular $\left|\phi_{o}(0)\right|^{2}=\|\omega\|_{L^{1}(\mathbb{D})}=1$. Also, polarizing, we obtain

$$
\phi_{o}\left(z_{1}\right) \overline{\phi_{o}\left(z_{2}\right)}=\int_{\mathbb{D}} \frac{1-z_{1} \bar{z}_{2}}{\left(1-\bar{w} z_{1}\right)^{2}\left(1-\bar{z}_{2} w\right)^{2}} \omega(w) d A(w) \quad\left(z_{1}, z_{2} \in \mathbb{D}\right) .
$$

Setting $z_{2}=0$, we find that $\phi_{o}=c(Q \omega)$, where $|c|=1$. Hence $Q \omega$ is outer, and substituting this back into (6.2) gives (6.1). This establishes (iii), and also shows that $\phi(z)=c z(Q \omega)(z)$.

Now we prove the 'if'. Suppose that $\omega$ satisfies (i), (ii) and (iii). Define $\phi(z):=z(Q \omega)(z)$. By (iii), the function $\phi$ belongs to the Smirnov class $N^{+}$, so it can be written as $\phi=b / a$ for some pair $(b, a)$. We claim that $\mathcal{D}_{\omega}=\mathcal{H}(b)$ with equality of norms. Property (i) implies that the norm $\|\cdot\|_{\mathcal{D}_{\omega}}$ is welldefined. Property (ii) implies that the Cauchy kernels $k_{z}(z \in \mathbb{D})$ span a dense subspace of $\mathcal{D}_{\omega}$ (as they do for $\mathcal{H}(b)$ ). It thus suffices to establish equality of norms, and by the same argument as in the proof of Theorem 4.1, it is enough to prove that $\mathcal{D}_{\omega}\left(k_{z}\right)=\left\|k_{z}^{+}\right\|_{H^{2}}^{2}$ for all $z \in \mathbb{D}$. This boils down to showing that (6.2) holds, which, with our definition of $\phi$, is equivalent to equation (6.1) of Property (iii). This establishes our claim and completes the proof.

The last theorem obviously begs the question as to which weights satisfy properties (i), (ii) and (iii). In particular:

Question 6.3. Which weights $\omega$ obey the relation (6.1)?

According to Theorem 4.1, equation (6.1) is satisfied by the weights

$$
\omega_{\zeta}(z):= \begin{cases}\frac{1-|z|^{2}}{|\zeta-z|^{2}}, & \zeta \in \mathbb{T}, \\ \log \left|\frac{1-\bar{\zeta} z}{\zeta-z}\right| \frac{2}{1-|\zeta|^{2}}, & \zeta \in \mathbb{D},\end{cases}
$$

as can also be verified by direct calculation. Are there any other solutions?

A possible source of examples are weights that can be expressed as the difference of two positive superharmonic functions. Such weights are given by the formula (2.2), where now $\mu$ is a finite signed measure on $\overline{\mathbb{D}}$. Part of the argument of the proof of Theorem 5.1 carries over to this case, showing that $\mu$ must still satisfy the moment relation (5.2). This raises another question:

Question 6.4. Which signed measures $\mu$ on $\overline{\mathbb{D}}$ satisfy the relation (5.2)?

Obviously this is the case if $\mu$ is a multiple of a Dirac measure. Are there any others?

\section{Acknowledgment}

Part of this research was carried out during a Research-in-Teams meeting at the Banff International Research Station (BIRS). We thank BIRS for its hospitality. 


\section{References}

[1] Aleman, A.: The multiplication operator on Hilbert spaces of analytic functions. Habilitationsschrift, Fern Universität, Hagen (1993)

[2] Chevrot, N., Guillot, D., Ransford,T.: De Branges-Rovnyak spaces and Dirichlet spaces, J. Funct. Anal. 259, 2366-2383 (2010)

[3] Costara, C., Ransford, T.: Which de Branges-Rovnyak spaces are Dirichlet spaces (and vice versa)? J. Funct. Anal. 265, 3204-3218 (2013)

[4] De Branges, L., Rovnyak, J.: Canonical models in quantum scattering theory. In: Perturbation Theory and its Applications in Quantum Mechanics (Proc. Adv. Sem. Math. Res. Center, U.S. Army, Theoret. Chem. Inst., Univ. of Wisconsin, Madison, Wis., 1965), 295-392, Wiley, New York (1966)

[5] De Branges, L., Rovnyak, J.: Square Summable Power Series. Holt, Rinehart and Winston, New York (1966)

[6] Duren, P.: Theory of $H^{p}$ Spaces. Dover, Mineola (2000).

[7] El-Fallah, O., Fricain, E., Kellay, K., Mashreghi, J., Ransford, T.: Constructive approximation in de Branges-Rovnyak spaces. Preprint.

[8] El-Fallah, O., Kellay, K., Mashreghi, J., Ransford, T.: A Primer on the Dirichlet space. Cambridge University Press, Cambridge (2014)

[9] Fricain, E., Mashreghi, J.: Theory of $\mathcal{H}(b)$ Spaces (vols 1 and 2). Cambridge University Press, Cambridge, to appear.

[10] Hedenmalm, H., Korenblum, B., Zhu, K.: Theory of Bergman Spaces. SpringerVerlag, New York (2000)

[11] Richter, S.: A representation theorem for cyclic analytic two-isometries. Trans. Amer. Math. Soc. 328, 325-349 (1991)

[12] Richter, S., Sundberg, C.: A formula for the local Dirichlet integral. Michigan Math. J. 38, 355-379 (1991)

[13] Sarason, D.: Sub-Hardy Hilbert Spaces in the Unit Disk. John Wiley \& Sons Inc., New York (1994)

[14] Sarason, D: Local Dirichlet spaces as de Branges-Rovnyak spaces. Proc. Amer. Math. Soc. 125, 2133-2139 (1997)

O. El-Fallah

Laboratoire Analyse et Applications (URAC/03), Université Mohamed V,

B.P. 1014 Rabat, Morocco

e-mail: elfallah@fsr.ac.ma

K. Kellay

Institut de Mathématiques de Bordeaux, Université de Bordeaux, 351 cours de la Libération, 33405 Talence Cedex, France

e-mail: karim.kellay@math.u-bordeaux1.fr

H. Klaja

Département de mathématiques et de statistique, Université Laval,

1045 avenue de la Médecine, Québec (QC), Canada G1V 0A6

e-mail: hubert.klaja@gmail.com 


\author{
J. Mashreghi \\ Département de mathématiques et de statistique, Université Laval, \\ 1045 avenue de la Médecine, Québec (QC), Canada G1V 0A6 \\ e-mail: javad.mashreghi@mat.ulaval.ca \\ T. Ransford \\ Département de mathématiques et de statistique, Université Laval, \\ 1045 avenue de la Médecine, Québec (QC), Canada G1V 0A6 \\ e-mail: ransford@mat.ulaval.ca
}

